\title{
From Symbiosis to SYM: Wider applications for biofeedback art
}

\author{
Kira Zhigalina \\ Artist, Deep Sym \\ Portobello road, \\ W11 London, UK \\ kirazhi@gmail.com
}

\author{
Ivan Isakov \\ Deep Sym \\ Darville road, \\ N16, London, UK \\ ivan@deepsym.com
}

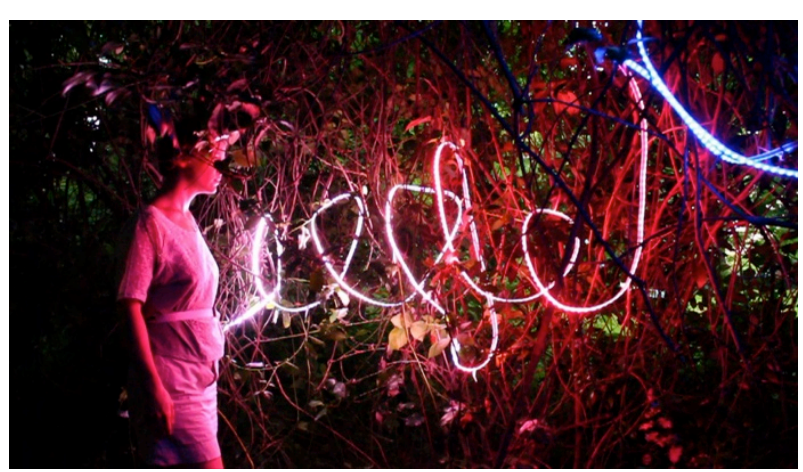

Figure 1: First Symbiosis tests with belt sensor

One of the later versions of Symbiosis is an immersive dome for 8 users. Each participant had a cushion with embedded sensors and was assigned different colour LEDs. When participants synchronised breathing, the lights grew out together from the top as one flower. The installation was designed to guide participants to use slow diaphragm breathing, through the location of sensors and software. The correct light response is only achieved with slowly increasing pressure from the lower back, which occurs by breathing deeply through the diaphragm. A gentle soundscape guided the breathing rhythm to be of 4 count inhale 6 count exhale, which is basic pranayama (ancient yogic breathwork practice).

\section{WIDER APPLICATIONS}

8.2 million people in the UK suffer from anxiety (Fineberg 2013), and medical research over the years has proven that one of the most effective techniques to deal with anxiety is deep diaphragm breathing. Conscious deep diaphragm breathing engages the cerebral cortex stimulating evolved areas of the brain, rebalances the autonomic nervous system, reduces heart rate and stimulates parasympathetic nervous system inducing calm and relaxed state (Varvogli 2011). 


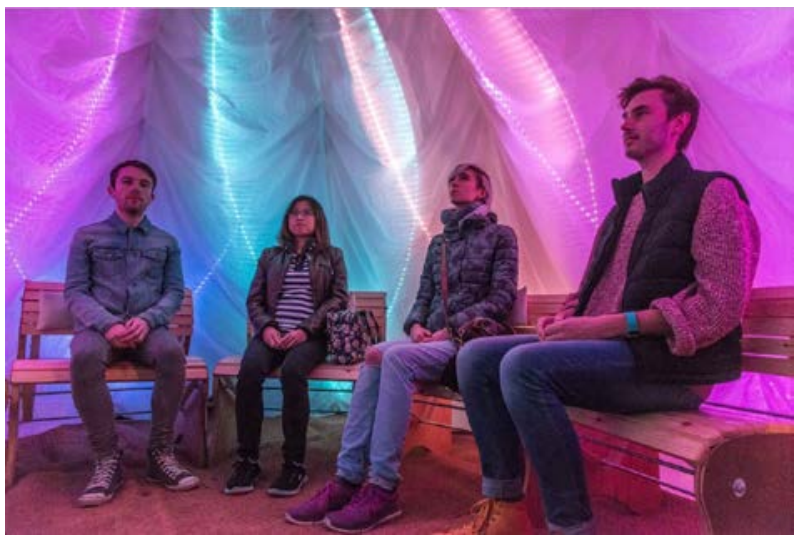

Figure 2: Inside Symbiosis at Leeds LightNight, UK

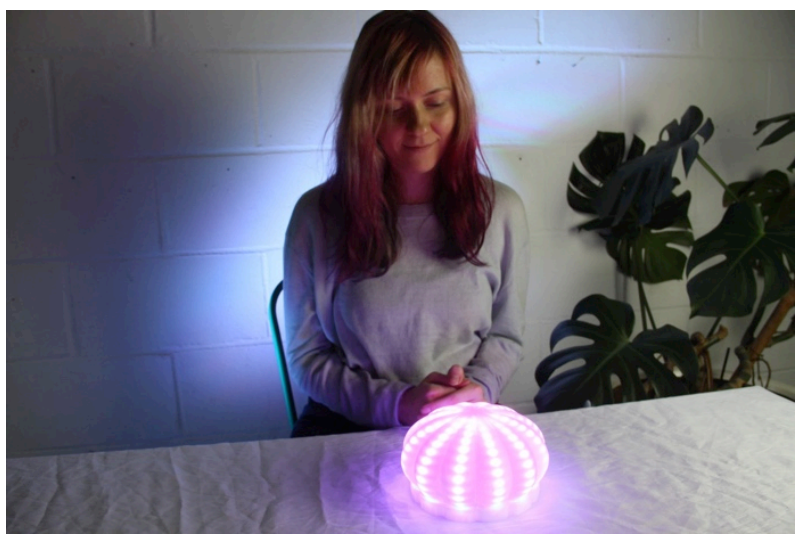

Figure 3: SYM user

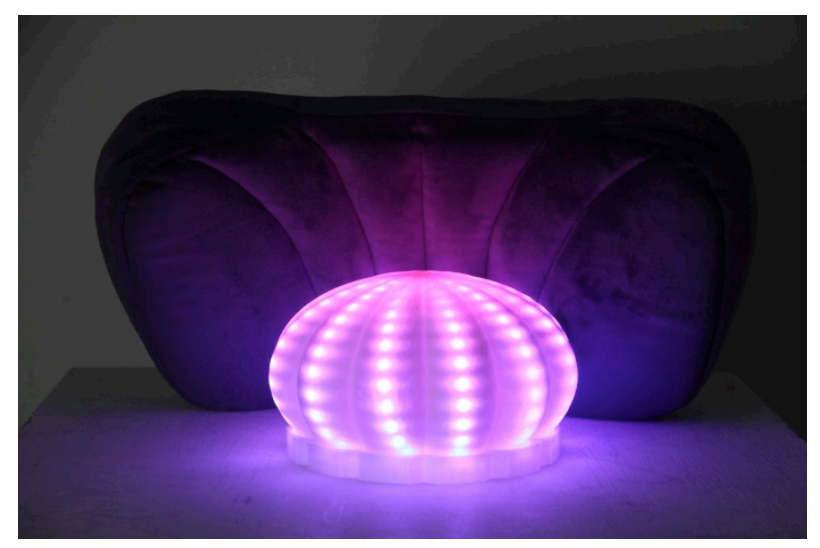

Figure 4: SYM light and sensor pillow
Showcasing Symbiosis has shown the need for the artwork to be experienced in a wider context beyond galleries, light and music festivals. In June 2019 we collaborated with Royal College of Music HEARTS study, which explores art, connectedness and mental health. We monitored the experience of the participants of Symbiosis at Great Exhibition Road Festival in Imperial College to a positive result of increased feeling of connectedness and mental wellbeing in $75 \%$ of the participants.

Participation in MedTech Supperconnector in 2019 resulted in collaboration with a gastroenterologist to apply Symbiosis concept to treat IBS patients through focus on deep diaphragm breathing to relieve symptomatic pain. This won the Best Pitch Award and resulted in creation of SYM, an art object that can be used in a home setting, creating ambience and calm through soothing lights that move with the user's breath, sensed through a custom pillow. A group of IBS patients were invited for a trial test. In the results of questionnaires $60 \%$ described the experience as excellent, and after $10 \mathrm{~min}$ use found their anxiety and gut related pain relieved.

Since then we have conducted more user research within informal settings and within psychotherapy practices. According to the psychotherapist, it could even be a tool to develop awareness and bring deep psychological changes. The potential of the effect of this adaptation of Symbiosis are worth investigating further and we would like to conduct more controlled research with groups of people who may be suffering from ailments such as anxiety, depression, stress or even asthma, using further biometrics such as heart rate and EEG to measure the effect of the participation on the levels of stress and anxiety.

\section{REFERENCES}

Fineberg, N., Haddad, P. M., Carpenter, L., Gannon, B., Sharpe, R., Young, A. H., Joyce, E., Rowe, J., Wellsted, D., Nutt, D. J., and Sahakian, B. J. (2013) The size, burden and cost of disorders of the brain in the UK. Journal of Psychopharmacology, 27(9), pp.761-770.

Varvogli, L., and Darviri, C. (2011) Stress Management Techniques: evidence-based procedures that reduce stress and promote health. Health Science Journal, 5(2), pp.74-81. 